\title{
Simulation and analysis of a meshed district heating network
}

\author{
Mattias Vesterlund, Andrea Toffolo, Jan Dahl \\ Department of Engineering Science and Mathematics, Division of Energy Science, Energy Engineering, \\ Luleå University of Technology, Luleå, Sweden, \{mattias.vesterlund; andrea.toffolo; jan.dahl\}@ltu.se
}

\begin{abstract}
:
The maturity of a district heating system can be estimated from its complexity. In fact, a mature network has a meshed structure while a younger network is often in the form of small network islands or has a tree structure. When a district heating system is developing and its complexity is increasing the flow distribution in the network is no longer obvious. As a consequence, the network owner, often the local energy company, is in need of a simulation program to have the possibility of analysing it and enlarge their network understanding. In this paper, a simulation tool developed in MATLAB/Simulink is applied in order to analyse the flow distribution in the district heating network of the town of Kiruna (Sweden). The Kiruna network has been developing since the 60 s and is today a mature network with the meshed structure. The method has specifically been developed to analyse the flow pattern in such kind of networks without altering their physical structure, and it is expected to be a valuable tool for the redesign of the network in the forthcoming relocation of some of the urban districts. The results about the current network configuration show that only a few pipes in the network are exceeding the recommended level of flow in terms of thermal power. The temperature and pressure drop from heat production site to the nodes serving the main consumer areas is within 1.6 bar and $10^{\circ} \mathrm{C}$ in the days of highest demand.
\end{abstract}

\section{Keywords:}

District heating, meshed network, simulation, flow pattern, heat distribution.

\section{Introduction}

District heating is a technology used for supplying a town district or a complete town with thermal energy generated in large heat production plants. By concentrating and scaling up the heat production instead of using single house boilers, a lower specific heat production cost can be obtained as well as a higher boiler efficiency and the possibility to co-generate electricity. Moreover, waste heat from nearby industries can be exploited as a suitable heat source by simply connecting the supplying facilities to the piping network. However, the thermal losses due the long range distribution of thermal energy must be taken into account, since 8 to $15 \%$ of the produced heat is lost in the pipes of a typical district heating network [1], [2].

The actual growth of district heating systems dates back to the period after the Second World War, when a large part of Europe had to be rebuilt [1]. The existing ones are often large and have an essential market share, as the outcome of a strong focus on the delivered quantity of thermal energy. More than 5000 district heating systems are in operation today within the European Union. The largest market shares, with over $40 \%$ of the building stock, are found in Denmark, Sweden, Finland, Poland, Estonia, Latvia and Lithuania [2], [3].

The history of Swedish district heating started in 1948 in Karlstad, the underlying energy policy being the reduction of the primary energy consumption by the concept of combined heat and power (CHP) generation [2]. Yet, the actual growth of the Swedish district heating technology occurred from the half of the $60 \mathrm{~s}$ to the half of $70 \mathrm{~s}$, when the governmental "Miljonprogrammet" was implemented [1]. The objective of the program was to create one million of new homes for families by building new city areas, which mostly consisted of apartment blocks [4]. These new areas had a significant heat demand density and no need for retrofitting, so it was natural to connect them to a district heating system [1], [2].

When a study is performed on the historical development of district heating networks, four different stages are found, which are shown in Figure 1 according to the evolution sequence: a) network with 
islands, b) coherent network with tree structure, c) network with ring, d) meshed network. In a simple network with a tree structure and only one heat production site the path of the hot water within the distribution pipes is univocally determined. In mature networks the structure tend to become meshed in order to reduce the heat load in the pipes. In a network with rings or meshes the hot water has a number of alternative paths from the heat production sites to the consumers, so that the impact of a line break can be reduced. In the same way the alternative paths within the network can be exploited when some parts of the system are undersized [1], [2].
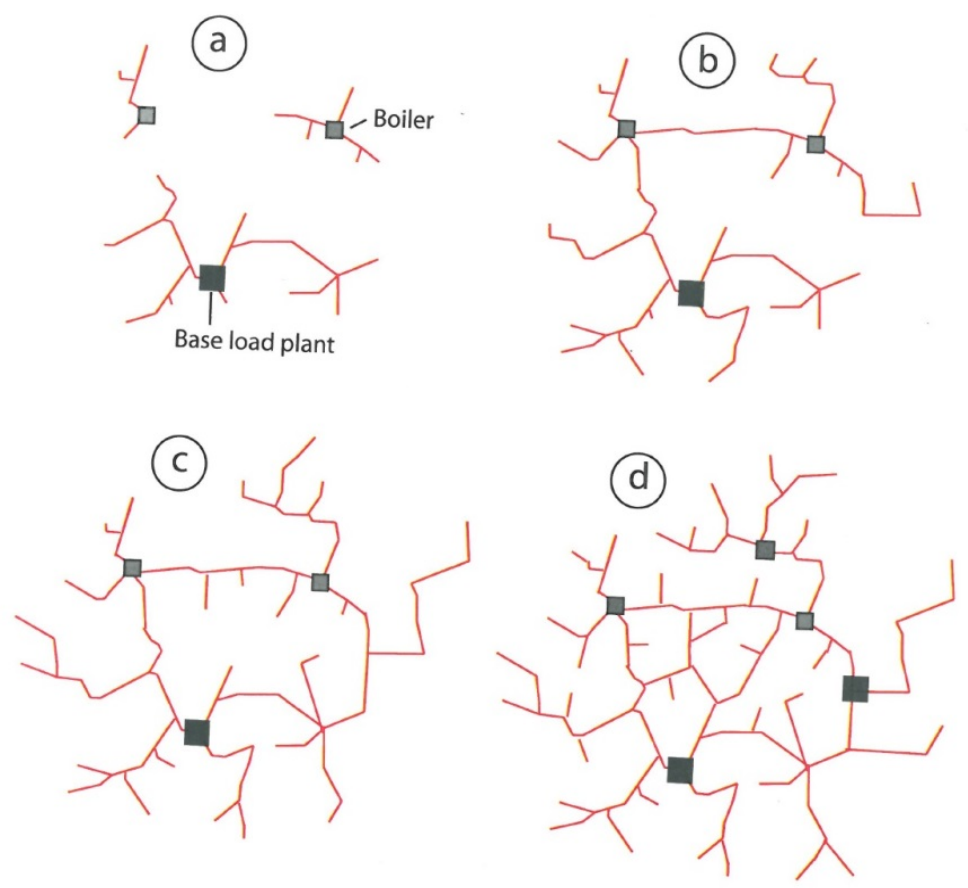

Fig. 1. Evolution of network designs, a) network with island, b) coherent network with tree structure, c) network with ring, d) meshed network [2].

The simulation and analysis of the hot water flow patterns in meshed district heating networks are not easy tasks because of the complexity of network structure. Consumer heat demands are of course the most important variables to determine flow patterns, but the energy company providing the heat delivery to the consumers has also two main concerns. The first is to guarantee that the temperature of the hot water supplied to the users is kept above a certain level. The second is that the pressure drop due to the flows in the pipes should be limited in order to keep the pumping power at the heat production sites within acceptable levels.

The literature about flow patterns in meshed networks offers a number of papers regarding electronic and computer networks [5], [6], [7], [8]. However, in the specific field of district heating few papers can be found. As far as optimization is concerned, the focus is on minimizing the pumping power in order to obtain a lower operating cost in the heat production sites [1], [2] or on the best mix of fuels [9], [10]. Other papers (e.g., [3], [11], [12]) concentrate on other aspects of heat production, even in multi-source networks, without discussing network behaviour. The methodology used in this paper is specifically conceived to analyse the flow pattern in meshed network, also with multiple heat production sites, and to visualize it together with pressure and temperature distributions.

\subsection{Case description}

Kiruna is a town in the north of Sweden (located $146 \mathrm{~km}$ above the Arctic Circle) close to the world's largest underground iron ore mine [13]. Deformation zones due to the mining activity have started to affect the town, which must now undergo a deep urban transformation in order to maintain the mining production. As shown in Error! Reference source not found. one third of the 
town is going to be affected by the subsidence in the next two decades, and the relocation of this part of the town has already been decided $2 \mathrm{~km}$ to the east [13], [14].

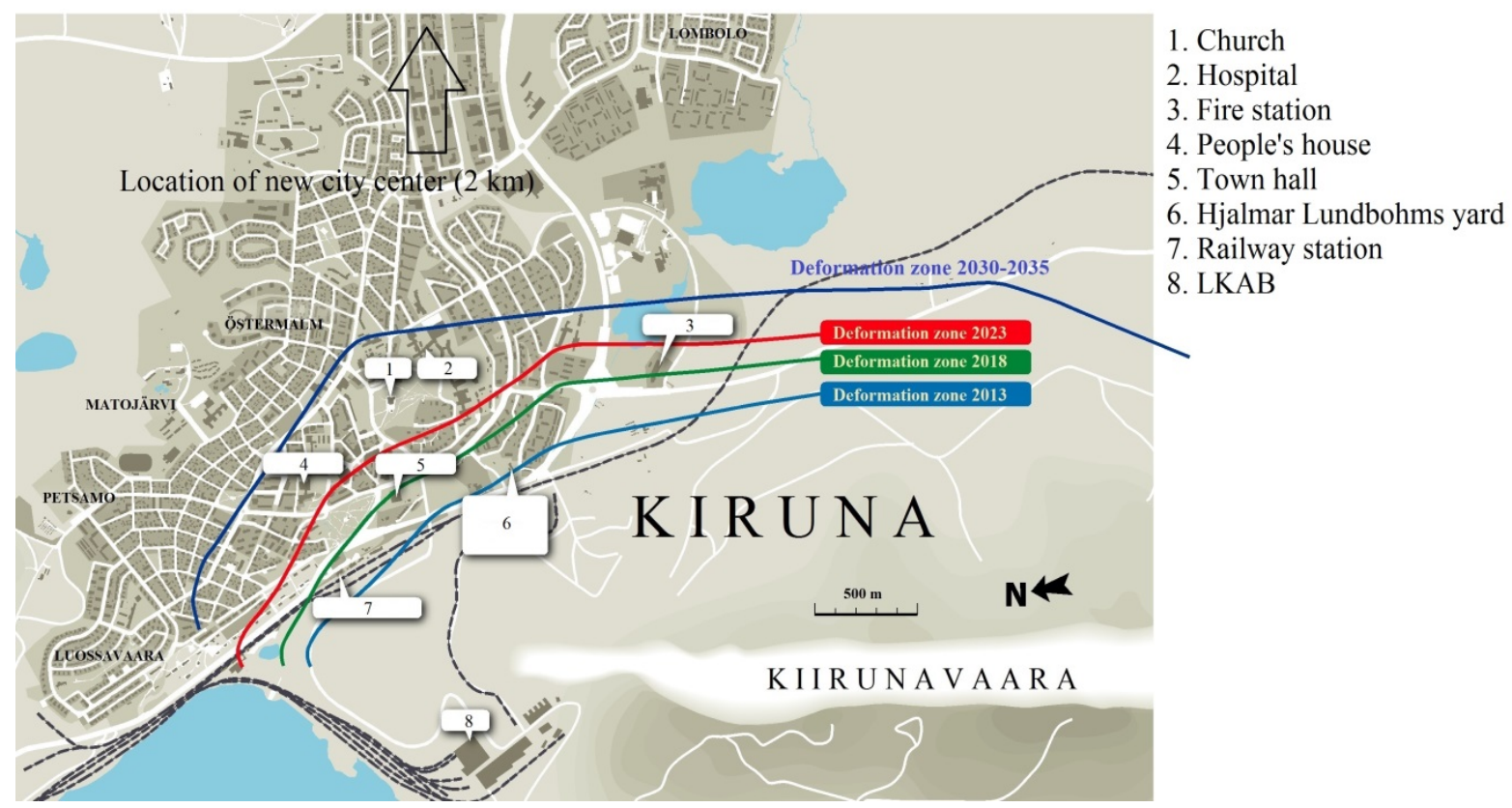

Fig. 2. The front of the subsidence affecting the town of Kiruna [13].

The infrastructures for the community must be reviewed and redesigned as a consequence of this urban transformation, and among them the district heating network, which provides thermal energy to the majority of the town buildings. The district heating network in Kiruna, owned by the local municipality company Tekniska Verken i Kiruna AB (TVAB), was started in 1963 and has been developed and expanded since then. Error! Reference source not found. shows the present structure of the district heating network in Kiruna. It represents a typical Swedish mature network structure in the meshed stage according to Error! Reference source not found., with heat production sites at different locations (multi-source network).

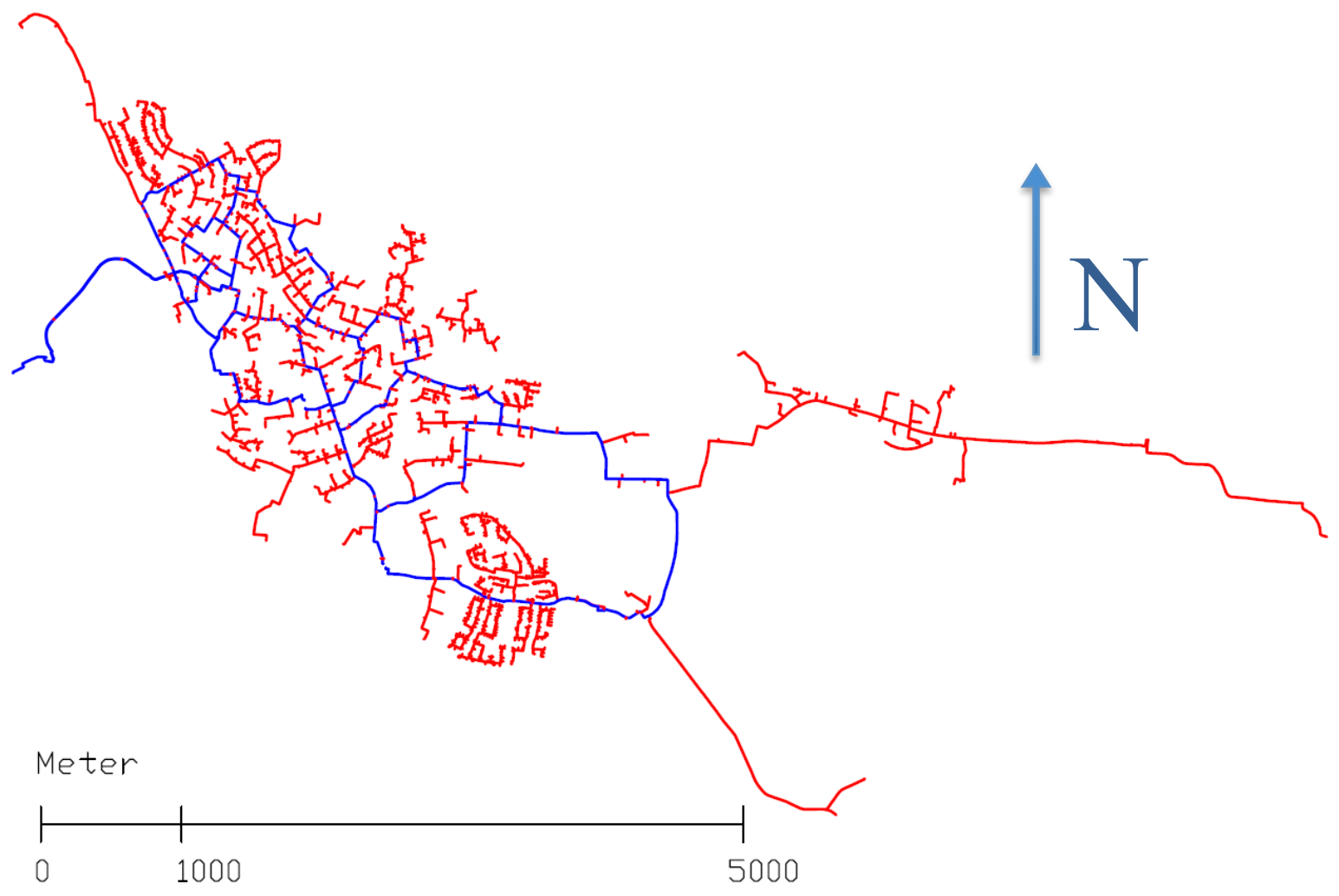


Fig. 3. The district heating network in the town of Kiruna: piping loops are shown in blue and consumer feeding branches in red.

The urban transformation of the town will require both an extension and some demolition of the current network. Before considering the redesign of a complex meshed network it is important to have a good knowledge about the behaviour of the current network, in order to be able to analyse how the expansions and the demolitions will affect the rest of the network. Optimized heat production is also important to obtain both for the current and the redesigned network.

The aim of this paper is to develop and simulate a model of the meshed district heating network for the town of Kiruna in order to:

- Analyse the flow pattern and find non-obvious paths in the network;

- Identify the critical pipes which are close to or exceed the recommended heat load;

- Obtain the temperature and pressure distribution in the network.

This information will be the basis for considering the possible alternatives in the future redesign of the network which is one of the fundamental tasks in planning the urban transformation. The tool used for this analysis is particularly suitable thanks to its modular structure that easily allow adding and removing network elements.

\section{Method}

This section describes the methodology used in the paper and is divided into two parts. The first describes the model developed in MATLAB/Simulink [15] to simulate the behavior of a meshed district heating network, which is also included in a broader process integration procedure for optimizing the operating cost of heat production in a multi-source network [16], while the second part is about the gathering of the required information to setup the model for the Kiruna case.

\subsection{The model of a meshed district heating network}

The nature of the meshed structure of the network requires a convenient model to simulate the distribution of the heat flows in the network piping. This is physically governed by the local pressure at piping nodes, which is therefore one of the fundamental variables that have to be calculated by the model. The other important aspect of the simulation is the evaluation of the heat losses in the network, since the temperature of the hot water reaching the consumers has decreased from its original supply value at the heat production sites. The temperature of the hot water at piping nodes is then the other fundamental variable that has to be calculated by the model.

The developed Simulink model reproduces the original structure of the network without any need of altering its multiple loop topology. The blocks in the diagram (see a detail of it in Figure 4) represent network pipes (red blocks), piping nodes (purple blocks) and the groups of final users (blue blocks).

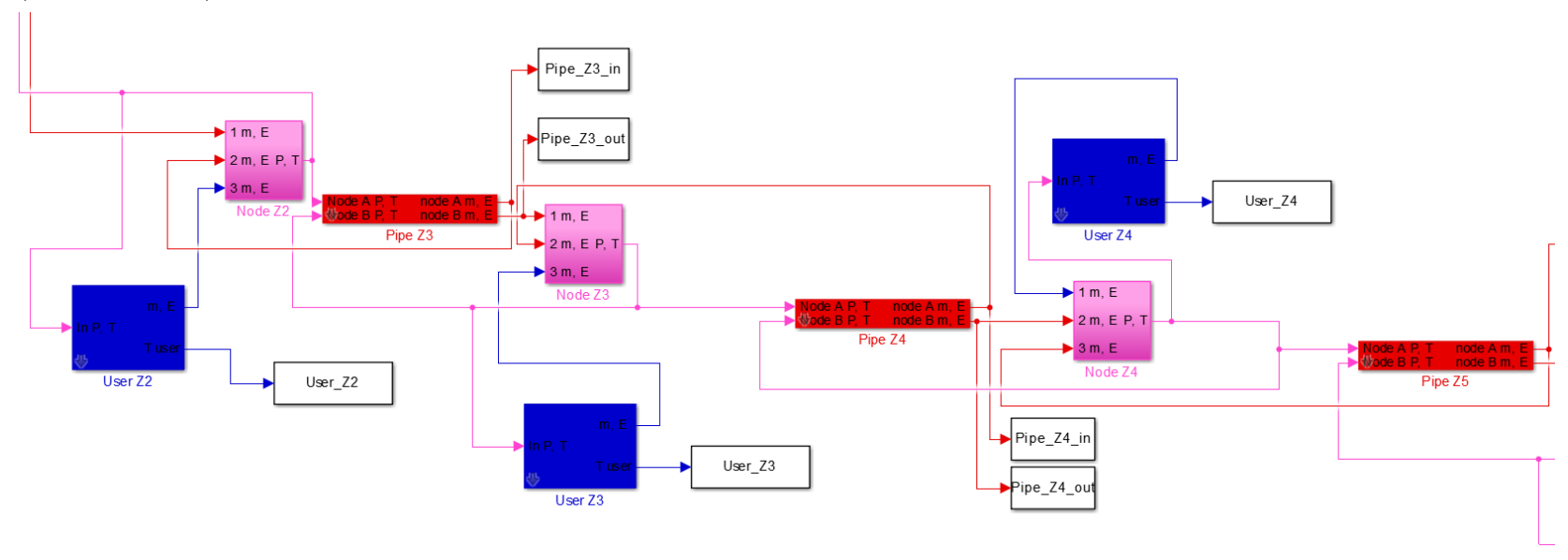


Fig. 4. Detail of the block diagram of the Simulink model for a meshed district heating network.

Piping node blocks represent the physical location of the junction between/among two or more different pipes of different diameter, and also the locations at which some thermal energy is extracted from the network loops by a consumer feeding branch. The pressure and temperature at each piping node are calculated using differential equations that express the mass and enthalpy balances in the block including all the incoming and outgoing flows (which are calculated in the pipe blocks). The mass and enthalpy unbalances are used to update the values of pressure and temperature at the node during the transient leading to the steady state behavior of the network

In the pipe blocks the mass flow rates and the associated enthalpy flows, which are affected by the thermal losses, are calculated. The mass flow rate $(\dot{m})$ is first determined as a function of the pressure drop $(\Delta \mathrm{p})$ through the pipe using Eq. 1. This requires the knowledge of the Darcy friction factor (f) that can be found as a standard equation in the literature, that is determined by the length $(\mathrm{L})$ and the diameter (D) of the pipe.

$$
\Delta p=f \frac{L}{D^{5}} \frac{8 \dot{m}^{2}}{\rho \pi^{2}}
$$

The associated enthalpy flow extracted from the upstream node $\left(\dot{Q}_{u p}\right)$ is evaluated as:

$$
\dot{Q}_{u p}=\dot{m} c\left(T_{u p}-T_{r e f}\right)
$$

where $c$ is the specific heat of water, $\mathrm{T}_{\text {up }}$ is the hot water temperature at the upstream node and $\mathrm{T}_{\text {ref }}$ is a reference temperature for the calculation of the enthalpy flows. The enthalpy flow that reaches the downstream node $\left(\dot{Q}_{\text {down }}\right)$ will be lower because of the heat losses in pipe $\left(\dot{Q}_{\text {loss }}\right)$,

$$
\dot{Q}_{\text {down }}=\dot{Q}_{u p}-\dot{Q}_{\text {loss }}
$$

These losses are considered to be proportional to the heat losses evaluated for the pipe in reference conditions $\left(\dot{Q}_{\text {loss,ref }}\right.$, for which a difference of $65^{\circ} \mathrm{C}$ between the average temperature of the water in the supply and return pipes $\left(T_{u p}\right.$ and $T_{\text {return }}$, respectively) and the ambient temperature $\left(T_{a m b}\right)$ is considered) according to the following equation:

$$
\dot{Q}_{\text {loss }}=\left(\frac{T_{u p}+T_{\text {return }}}{2}-T_{\text {amb }}\right) \frac{\dot{Q}_{\text {loss }, \text { ref }}}{65}
$$

Since the quantities calculated in one type of blocks are needed by the other type of blocks and vice versa, a critical exchange of information must occur between pipe and node blocks (pipe mass and enthalpy flows vs. node pressure and temperature). It is therefore apparent that the fundamental structure of the model relies on the strict alternation of pipe and node blocks along the representation of the meshed network topology.

The conditions at the boundaries of the district heating network model are of two types. At the sources, i.e. the heat production sites, the pumping pressure and the supply temperature must be specified. At the sinks, i.e. the final users, the thermal demand of the group of consumers $\left(\dot{Q}_{\text {demand }}\right)$ must be specified, but also the thermal losses in the area served by the corresponding feeding branch have to be taken into account $\left(\dot{Q}_{\text {loss, area }}\right)$. The mass flow rate $\dot{m}_{u s e r}$ extracted from the node (at temperature $T_{\text {node }}$ ) from which the feeding branch departs is calculated as

$\dot{m}_{\text {user }}=\frac{\dot{Q}_{\text {demand }}+\dot{Q}_{\text {loss, area }}}{c\left(T_{\text {node }}-T_{\text {return }}\right)}$

where the thermal losses in the area are obtained by using Eq. 4 and reference loss for the area. In this way the mass flow rates extracted from the network by the users depend not only on the demand of the groups of consumers, but also on the temperature of the hot water at the node in which the extraction takes place (which is in turn a function of the thermal losses along the network and the distribution pattern of the thermal energy). 


\subsection{Model setup for the Kiruna case}

The data required to build a model that is specific for the Kiruna case have been mainly provided by local municipality company (TVAB) through a geographical information system (GIS) map. This is a virtual map of the network in which information can be stored [16]. The data extracted from the GIS are related to:

- The location, the length and the diameter for each pipe in the network. Pipe diameters are then used to determine the friction coefficient and the reference thermal losses of the pipes from manufacturer data. Some lack of data about pipe diameters has been fixed with a statistical strategy described in details in [17].

- The classification of pipes into those belonging to the loops of network topology and those belonging to the consumer feeding branches (see Figure 3).

- Consumer heat consumption on a daily basis. Consumers are grouped into user areas according to the main feeding branches departing from the network loops.

- The supply temperatures at the heat production sites on a daily basis (in this paper only one heat production site is considered, the main TVAB facility, and a reference pressure of 0 bar is assigned to it as pressure boundary condition).

\section{Analysis of the results}

The model of the meshed district heating network in Kiruna has been simulated on a daily basis in the months of January and February 2010. These are usually the coldest winter months and during this period the heat demand from the network users is the highest, so the highest thermal energy flows are expected to occur in network piping. In order to study and analyze the network behavior a graphical interface was created in MATLAB, which reads the result generated from the simulation and allows to visualize them in the ways that are presented in the following.

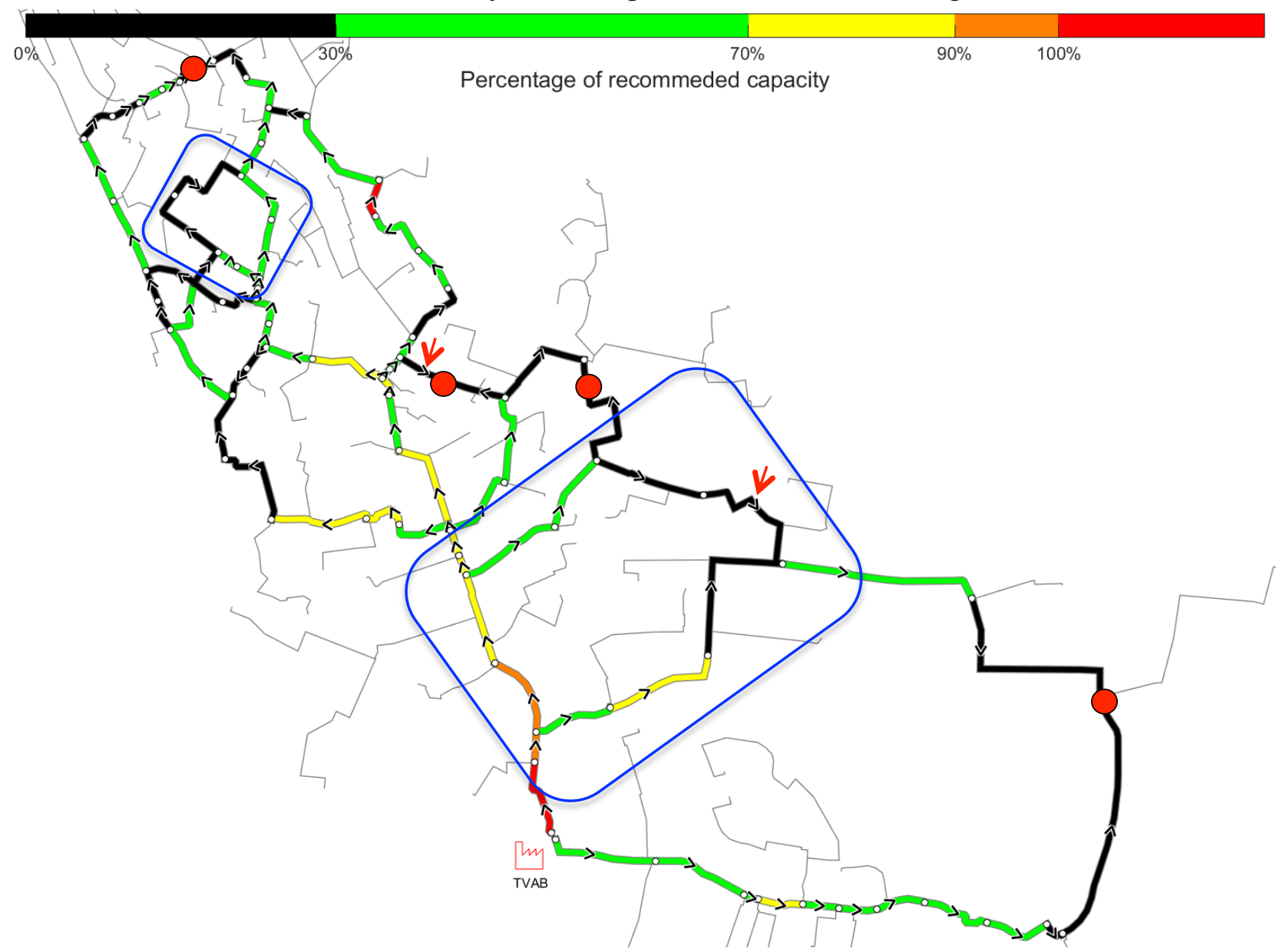

Fig. 5. Flow pattern for a typical winter day in the district heating network of Kiruna. 


\subsection{Flow pattern of a meshed network}

The visualization of the pattern of the thermal energy flows in the network is shown in Figure 5 for a typical day in the simulated period. The different colors are used to represent the value of the ratio between actual amount of the thermal energy flow in the pipe and the recommended pipe capacity as specified by the manufacturer [18]. Black arrows are used to show the direction of the flow in the pipes: the pattern of flow directions does not change at all in the simulated days except for the two pipes pointed by the red arrows (in these pipes the flow direction is the opposite in just one of the days). The nodes that do not have any outgoing flow (except of course the one to the served user area) are marked with red circles.

\subsection{1. "Non-obvious" paths}

The typical flow pattern in the network shows that there are two different sections in which the thermal energy delivered to a user area is actually transported through a longer route than needed. These "non-obvious" paths (marked with blue rectangles in Figure 5) are shown in Figure 6. In fact, in each section two alternative paths are used according to the flow direction arrows in order to reach the node marked in red from the node marked in blue. If only the shortest alternative were used, the route for the delivery of the heat could be reduced by 466 and 908 meters respectively. It is likely that the temperature drop along the longer path will be higher because of higher heat losses and the lower (split) mass flow rate associated with the thermal energy flow, so that the temperature in the red node will be lower once the hot water from the longer and the shorter paths is mixed.

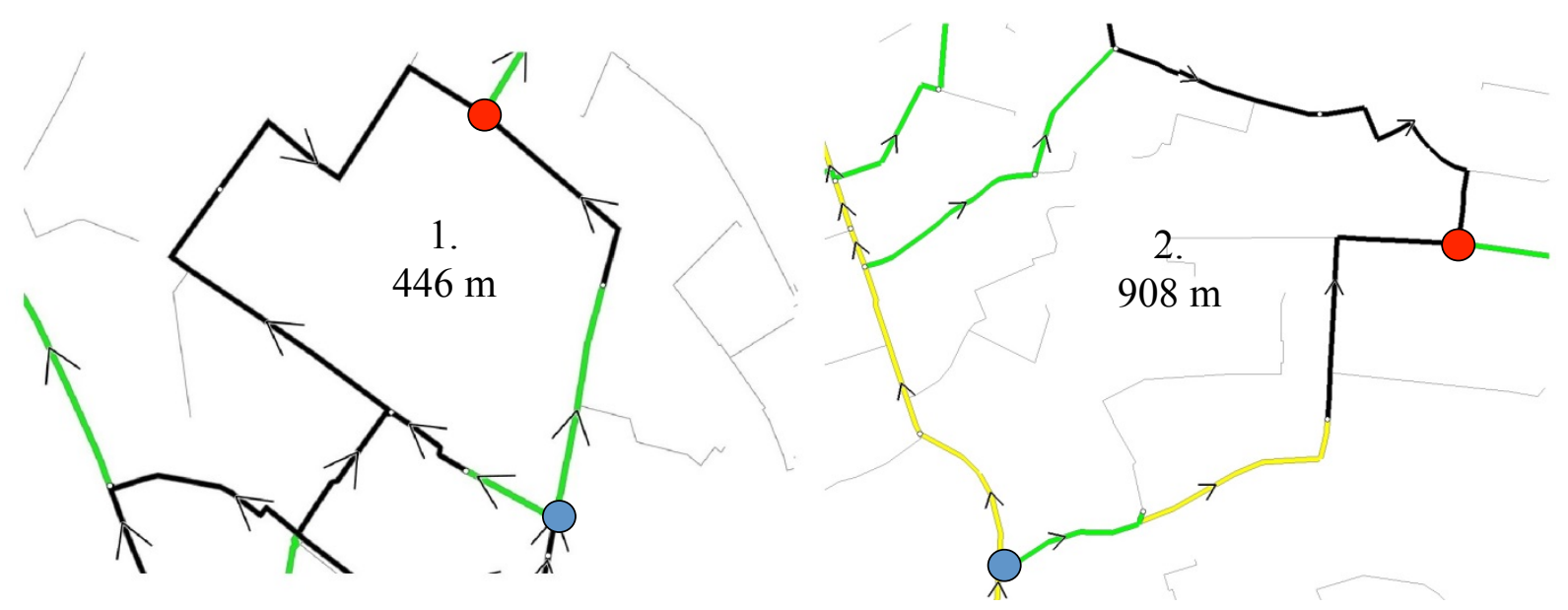

Fig. 6. Detail of the non-obvious paths in the network from Figure 5.

\subsubsection{Critical pipes}

The visualization in Figure 5 allows one to identify the critical pipes that are close to or exceed the capacity recommended by the manufacturer. In the 59 simulated days only two pipes exceeds the recommended thermal flow and are those already shown in red in Figure 5. The first is the main pipe that starts from the TVAB heat production site and convoys a large share of the generated hot water to the north (the current location of the city center). The second one is a generic pipe in one of the network loops that apparently must have been undersized.

The ratios between the actual thermal energy flow and the recommended one during the simulated period are shown in Figure 7 for all the pipes that exceed a ratio of $70 \%$ (yellow color in Figures 5 and 7) for at least one day.

In addition to the two mentioned red pipes, there are two pipes that reach a ratio of $90 \%$ (orange color in Figures 5 and 7) in the days characterized by the highest loads. As shown in Figure 5, these are again located along the "backbone" of the network that convoys the hot water from the TVAB heat production site to the northern districts of the town. 


\section{Ratio between actual and recommended thermal power in the pipes}

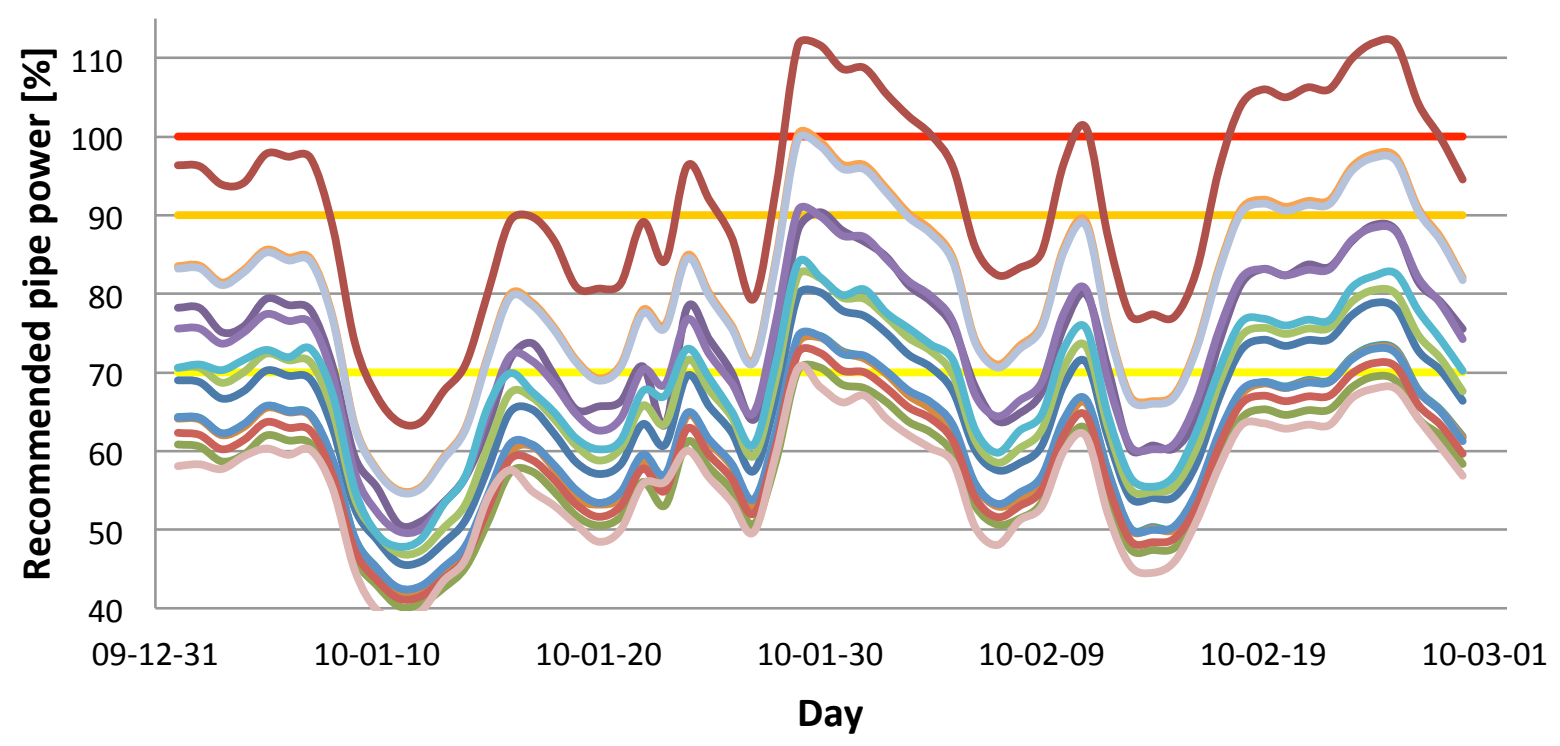

Fig. 7. Daily thermal energy flow in the simulated period for the pipes exceeding $70 \%$ of recommended capacity for at least one day.

\subsection{Temperature and pressure distributions}

The temperature and pressure distributions can be seen in Figure 8 and 9, respectively, for the day in which the heat load in the network was the highest.

Figure 8 shows both the temperature distribution in the meshed part of the network (the colors in the nodes and in the piping loops) and the average temperatures of the hot water supplied to the user areas (the colors in the areas enclosing the main feeding branches). Of course the highest temperature is the supply temperature at the TVAB heat production site $\left(94.8^{\circ} \mathrm{C}\right)$. The maximum temperature drop within the meshed part of the network is lower than $10^{\circ} \mathrm{C}$. The average temperature of some of the user areas is however lower (even if they are closer to the TVAB facility). The reasons for a marked reduction of the hot water temperature are the high heat losses accumulated along the route towards a far node/area or mass/enthalpy flows that are too low with respect to the heat losses in the pipe or in the user area, or both.

Figure 9 shows the pressure distribution in the meshed part of the network (the colors in the nodes and in the piping loops) starting from the TVAB heat production site that has been assigned a reference pressure of 0 bar. It is apparent that the maximum pressure drop in the network (about 1.6 bar in the day of the highest thermal demand) is registered in the farthest nodes in the northern districts of the town.

\section{Discussion}

The district heating network in Kiruna has the structure that is typical of mature network in the last stage of their evolution (meshed networks) and the analysis of the flow pattern confirms the benefit stated in the introduction about distributing the thermal energy through several pipes instead of one. A couple of sections with non-obvious paths are also found, which are an inherent drawback of these type of network topologies. 

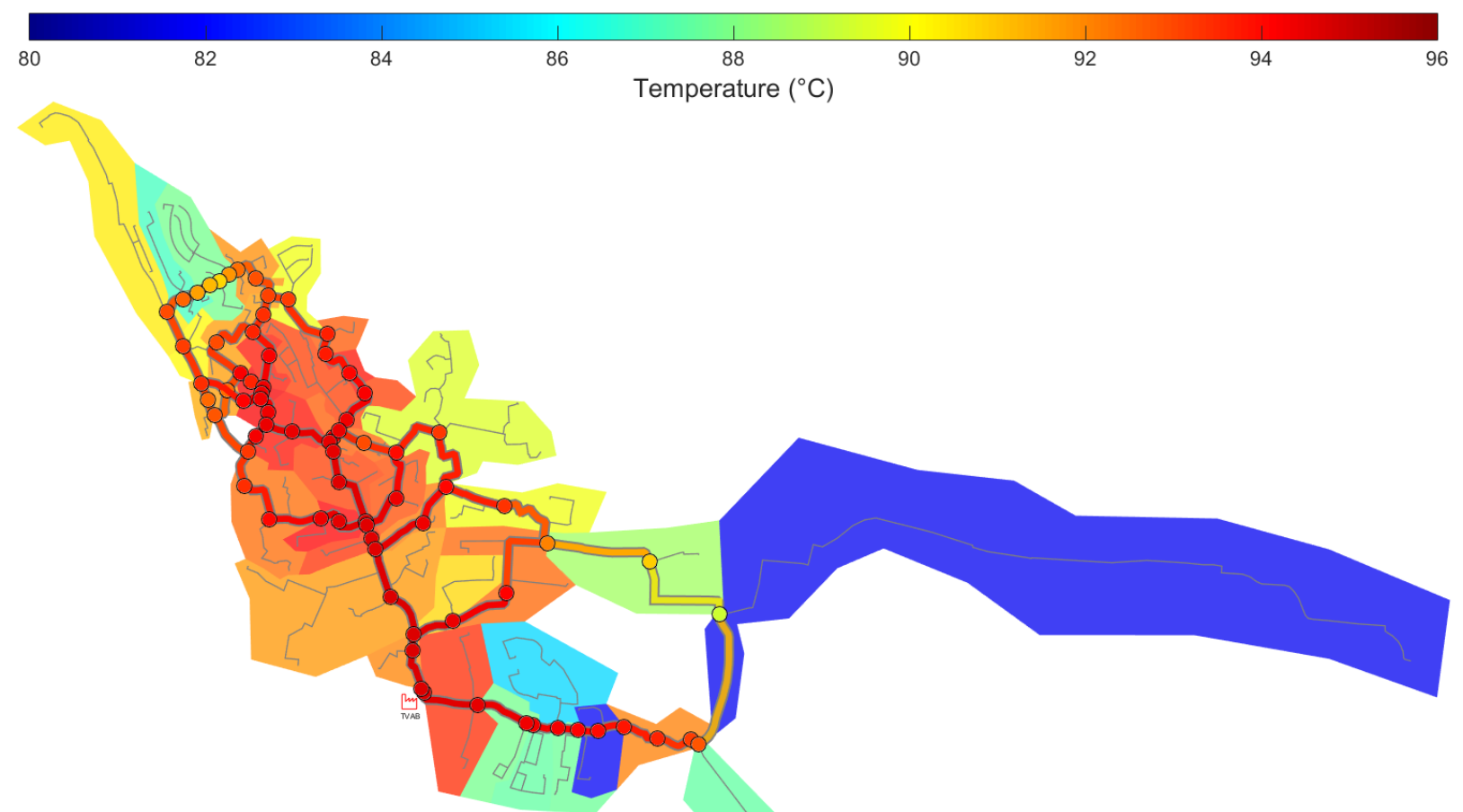

Fig. 8. Temperature distribution for the meshed part of the district heating network and the user areas.

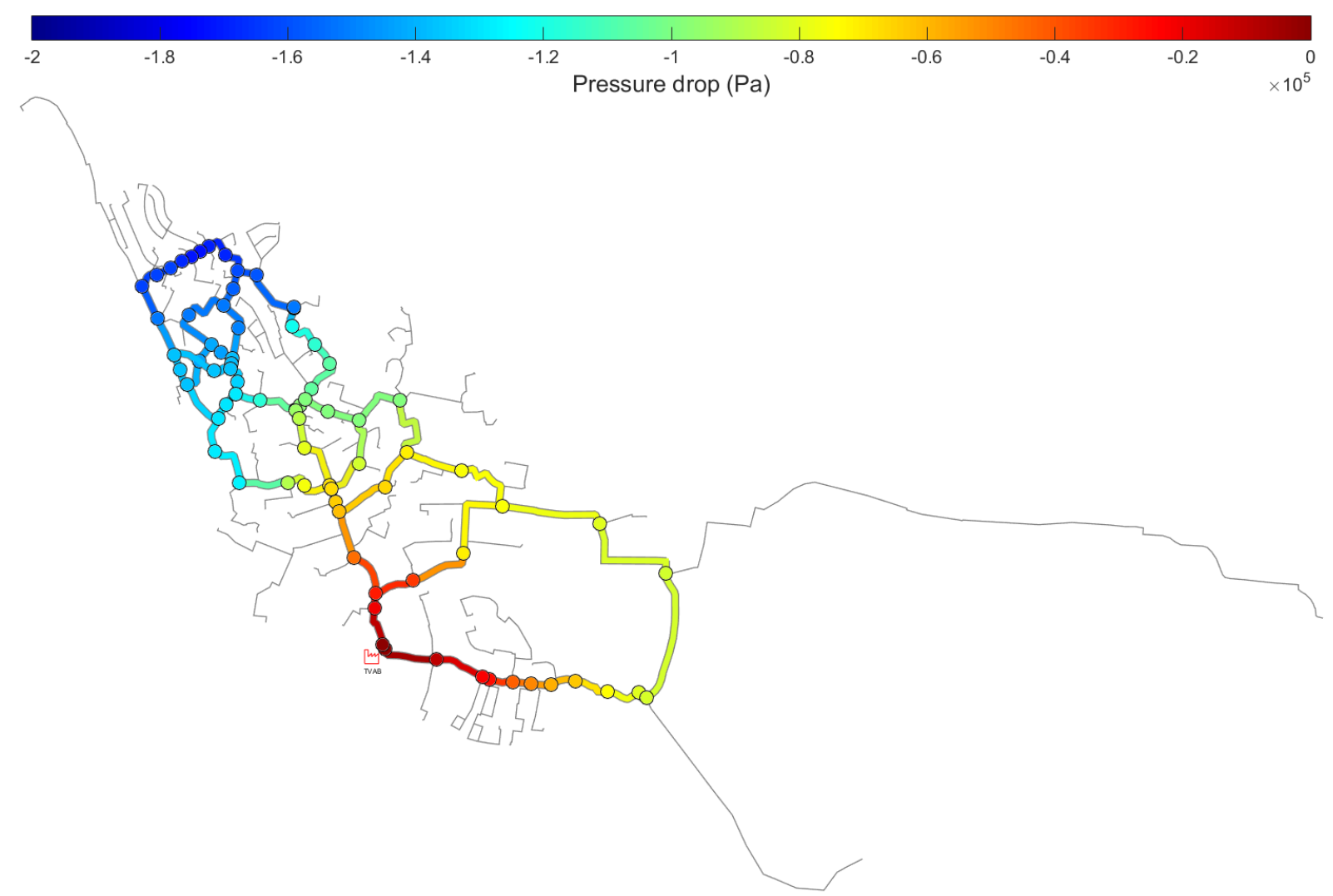

Fig. 9. Pressure distribution for the meshed part of the district heating network (TVAB heat production site is assigned a 0 bar reference pressure). 
The ratios between the actual thermal energy flows in the pipes and the recommended ones show that the current network is generally oversized. The few pipes that are close to or above the recommended capacity in the days of the highest demand are the pipes departing from the TVAB heat production site along the "backbone" delivering heat to the northern districts of the town, that is those that will be relocated due to the urban transformation. Only one isolated pipe in a secondary network loop seems to be a minor undersized bottleneck. The size of the current network therefore allows for margins of expansion, in particular towards the areas east of the town where the new district will be built.

The pattern of flow directions was found to be almost completely insensitive to the variation of thermal loads in the meshed part of the network. This is probably due to the time resolution of the simulations, which were performed using a daily basis. A finer (e.g., hourly) resolution may reveal that inversions of flow direction occur in higher number of pipes and/or more frequently.

The local values of pressures and temperature obtained with the model show a fairly good agreement with real ranges of pressure and temperature drops along the meshed part of the network. However, a detailed comparison between the real and the simulated values is not possible at this stage of model development because a constant temperature has been considered for the return pipes all through the network.

The developed model has proved to be suitable for the analysis of the flow pattern in district heating network with a complex meshed structure. Thanks to the modularity in the configuration of the block diagram, it will be useful for investigating how the network is affected by the addition of other heat production sites (multi-source network). It will also be appropriate to use it in the redesign of the network due to the urban transformation in Kiruna, when new loops will be added to the meshed piping structure and part of the current network will be abandoned.

\section{Conclusions}

The tool developed in MATLAB/Simulink is a valuable instrument to simulate and analyse the behaviour of meshed district heating networks. Flow distribution as well as pressure and temperature drops along network pipes can be reproduced and visualised for identifying critical sections. The strategy of using a small number of predefined blocks make the models fully modular, so that the tool can be applied to different levels of network complexity. Another fundamental strength is that the way in which the predefined block are connected and the operation they perform prevents the necessity of introducing artificial modification to the original structure.

The district heating network in Kiruna is a mature network featuring several loops and will have to be redesigned in the near future to comply with a major urban transformation. This work was conceived as basic study on the behaviour of the current Kiruna district heating network and as a preliminary step towards the multidisciplinary investigation involved in its redesign.

The simulation of the current network behaviour shows that:

- Few pipes have a peak load that is close to or exceeds the recommended heating capacity. All except one belong to the backbone of the network, the load which will be heavily reduced when the northern districts of the town will be relocated;

- The directions of the water flow inside the pipes are stable in a daily basis analysis;

- In a couple of loops the heat is transported through a path that is longer than necessary, but this is an inherent drawback of meshed networks;

- The temperature and pressure drops in the meshed part of the network are within acceptable ranges (less than 1.6 bar and $10^{\circ} \mathrm{C}$ during the days of maximum load);

- While the meshed part of the network has a smooth temperature distribution, large temperature drops occurs in the feeding pipes to the users, so that consumer areas may be supplied with water having an average temperature which is quite lower than that at the nodes supplying them. 


\section{Acknowledgments}

This work is founded by HLRC (Hjalmar Lundbohm Research Centre) with is a sponsored by local mining organisation LKAB and by the ATTRACT (Attractive, sustainable habitats in cold climates) that is a research program financed by VINNOVA (Sweden's Innovation agency).

\section{References}

[1] S. Frederiksen, S. Wener, Fjärrvärme - Teori, teknik och funktion, Studentlitteratur, Lund, 1993.

[2] S. Frederiksen, S. Wener, District Heating and Cooling, Studentlitteratur, Lund, 2013.

[3] M. Åberg, J. Widén, Development, validation and application of a fixed district heating model structure that requires small amounts of input data, Energy Conversion and Management 75 (2013) 74-85.

[4] J. Jörnmark, Nationalencyklopedin - Miljonprogrammet 2014.

[5] S. De, C. Qiao, A hybrid meshed multipath forwarding scheme in wireless ad hoc networks, Comput. Commun. 30 (2007) 3346-3357.

[6] S. De, C. Qiao, H. Wu, Meshed multipath routing with selective forwarding: an efficient strategy in wireless sensor networks, Computer Networks 43 (2003) 481-497.

[7] H. Li, A. Zhang, X. Shen, J. Xu, A load flow method for weakly meshed distribution networks using powers as flow variables, International Journal of Electrical Power \& Energy Systems 58 (2014) 291-299.

[8] D. Rajičić, R. Taleski, Two novel methods for radial and weakly meshed network analysis, Electr. Power Syst. Res. 48 (1998) 79-87.

[9] M. Burer, K. Tanaka, D. Favrat, K. Yamada, Multi-criteria optimization of a district cogeneration plant integrating a solid oxide fuel cell-gas turbine combined cycle, heat pumps and chillers, Energy 28 (2003) 497-518.

[10] E.S. Barbieri, Y.J. Dai, M. Morini, M. Pinelli, P.R. Spina, P. Sun, R.Z. Wang, Optimal sizing of a multi-source energy plant for power heat and cooling generation, Appl. Therm. Eng.

[11] V. Vittorio, B. Giorgia, Primary energy reductions in district heating networks through variation of the thermal load profile ot the users, Proceeding of 27th International Conference on efficiency, Cost, Optimization, Simulation and Enviromental Impact of Energy Systems (2014).

[12] D. Chinese, Optimisation models for decision support in the development of biomass-based industrial district-heating networks in Italy, Appl. Energy 82 (2005) 228-254.

[13] LKAB, LKAB Homepage 2014.

[14] Kiruna Kommun, The Urban Transformation 2014.

[15] Matlab, Matlab Simulink 2014. 
[16] M. Vesterlund, J. Dahl, A method for the simulation and optimization of district heating systems with meshed networks, Energy Conversion and Management 89 (2015) 555-567.

[17] M. Vesterlund, S. Johan, B. Lindblom, J. Dahl, Evaluation of losses in district heating system, a case study, Proceeding of 26th International Conference on efficiency, Cost, Optimization, Simulation and Enviromental Impact of Energy Systems (2013).

[18] Powerpipe, Pipe specifications (2011). 